Cinémas

Revue d'études cinématographiques

Journal of Film Studies

\title{
Les Mots de la tribu
}

\section{Gilles Marsolais}

Volume 4, numéro 2, hiver 1994

\section{Le Documentaire}

URI : https://id.erudit.org/iderudit/1001027ar

DOI : https://doi.org/10.7202/1001027ar

Aller au sommaire du numéro

\section{Éditeur(s)}

Cinémas

\section{ISSN}

1181-6945 (imprimé)

1705-6500 (numérique)

Découvrir la revue

\section{Citer cet article}

Marsolais, G. (1994). Les Mots de la tribu. Cinémas, 4(2), 133-150. https://doi.org/10.7202/1001027ar

\section{Résumé de l'article}

Les années 80 ont été fertiles en tentatives de dépassement des clivages traditionnels entre le film documentaire et le film de fiction, selon un authentique processus de "pollinisation ». Aussi, on a tort de vouloir considérer l'ensemble des films issus de ce phénomène sous l'appellation générique «docu-fiction »; ce nivellement a le défaut d’occulter la richesse et la diversité d'un ensemble de pratiques significatives, sinon révélatrices des " maux de l'attribut » : fiction ou documentaire? À l'aide d'exemples tirés du cinéma québécois, ce texte revient sur la terminologie et propose une classification plus fine des modalités de cette " pollinisation », en prenant en compte le point de vue du spectateur, ce nouveau " primitif " à travers lequel se profile peut-être " la tribu du troisième type ». 


\section{Les Mots de la tribu}

\section{Gilles Marsolais}

\section{RÉSUMÉ}

Les années 80 ont été fertiles en tentatives de dépassement des clivages traditionnels entre le film documentaire et le film de fiction, selon un authentique processus de "pollinisation". Aussi, on a tort de vouloir considérer l'ensemble des films issus de ce phénomène sous l'appellation générique "docu-fiction"; ce nivellement a le défaut d'occulter la richesse et la diversité d'un ensemble de pratiques significatives, sinon révélatrices des "maux de l'attribut" : fiction ou documentaire? À l'aide d'exemples tirés du cinéma québécois, ce texte revient sur la terminologie et propose une classification plus fine des modalités de cette "pollinisation", en prenant en compte le point de vue du spectateur, ce nouveau "primitif" à travers lequel se profile peut-être «la tribu du troisième type».

\section{ABSTRACT}

The 1980s saw a profusion of attempts to get beyond the traditional division between documentary and fiction films in a veritable process of "cross-pollination." But it would be misleading to classify all the resulting films in the genre of "docu-fiction." Such reductionist labelling would obscure the richness and the diversity of their signifying practices and reveal only the irresistible impetus to categorize as fiction or documentary. Using examples drawn from Québécois cinema, this text reexamines the relevant terminology and proposes a more finely-grained classification of the forms that this crosspollination has taken, one that takes into account the point of view of the spectator, that "new primitive" who can be thought to represent a "tribe of the third type." 
"C'est un grand moment de la vie d'un peuple que celui où tout le monde, ou presque tout le monde s'applique à employer les mots dans leur sens véritable; et c'est un moment terrible de cette vie, quand, à nouveau, ceux qui avaient cessé de le faire, se remettent à jouer avec ces mots...”

Louis Aragon, Servitude et Grandeur des Français

Comme le laisse soupçonner le titre même de cet article emprunté à Mallarmé ${ }^{1}$ qui, en son temps, déjà, proposait de "Donner un sens plus pur aux mots de la tribu...", cette citation jetée là d'entrée comme un "Calme bloc ici-bas chu d'un désastre obscur" concerne tout à fait l'homo cinematographicus.

Ainsi en est-il de cet aphorisme : "À vouloir faire vrai, le cinéma sort toujours perdant." Aussi largement répandu qu'il est d'une banalité affligeante, il dit tout et rien du tout à la fois, puisqu'il parle à la fois de tout et de rien. Certains critiques ou analystes s'en servent pour dénoncer à juste titre les efforts considérables consacrés dans certains films à la reconstitution exhaustive d'une réalité ou d'un événement. La lourdeur stylistique qui résulte généralement d'une telle application condamne à elle seule et sans appel ce type de pratique, cette volonté de "vouloir faire vrai». Le cinéma américain est prodigue de ces monuments d'ennui, généralement d'une durée supérieure à la moyenne, qui semblent produits davantage pour faire vendre du "pop corn" ${ }^{2}$ que pour provoquer un choc artistique, une rencontre du spectateur avec un film ou avec un auteur. Le fractionnement du travail et la spécialisation des divers métiers du cinéma ne sont pas étrangers à ce phénomène qui a pris de l'ampleur au cours des dernières années. Rares sont les cinéastes, indépendants ou européens, qui peuvent encore échapper peu ou prou à ce moule de fabrication, qui font exception et qui nous réservent à l'occasion l'un de ces chocs s'apparentant à quelque "rencontre du troisième type», tant leurs films, qui prennent leurs distances face cette pratique de l'imitation ou de la reconstitution maladroite, semblent sortir de l'ordinaire de la consommation cinématographique.

Dans la foulée, on est tenté d'établir un rapprochement avec certaines reconstitutions historiques du cinéma des premiers temps. Mais, à la double différence que celles-ci se présentaient 
généralement comme des "actualités" et qu'elles étaient ou pouvaient être lues comme telles par le spectateur naïf de l'époque (la lecture qu'il faisait des Méliès était fort différente), alors que le spectateur d'aujourd'hui, devant les produits de consommation d'inspiration américaine qu'on lui propose, "sait" qu'il assiste à une reconstitution, et que son plaisir consiste plutôt à vérifier l'énumération et la conformité des diverses plages d'une biographie ou à évaluer (Hoffa: 5/10; Chaplin : 8/10) les modalités de cette évocation (comme l'épaisseur du maquillage, l'embonpoint volontaire de l'acteur, etc.), quand l'omniprésence d'une musique simpliste ne réussit pas à neutraliser ses quelques neurones encore actifs.

Précisons aussi que ces "actualités" reconstituées du cinéma des premiers temps, prétendants ingénus (?) d'un "genre» à naître, n'avaient de fait rien à voir avec ce que l'on nommera bientôt «le documentaire». Leur projet relève de la fiction pure ${ }^{3}$, alors que les déviances ou les écarts de celui-ci sont d'un autre ordre.

D'autres critiques ou analystes ont aussi recours à cet aphorisme pour dénoncer à tort le documentaire, comme si le "genre" était univoque, et même le substantif qui tente de cerner la réalité théorique et pratique qu'il désigne. Il ne fait aucun doute que certains soi-disant "documentaristes» trichent sans vergogne avec la réalité approchée par les moyens du cinéma et avec le matériau filmique (les entreprises du genre Mondo Cane ont suscité une progéniture qui s'exprime aujourd'hui surtout dans le cadre de certaines émissions télévisées) : ils ont tôt fait d'être démasqués par le spectateur dont les neurones ne sont pas trop atteints, et de se discréditer en tant que documentaristes. D'un point de vue théorique, osons le rapprochement, leurs films se voient en quelque sorte ramenés au statut de ces bandes filmiques d'un certain cinéma "d'actualités" des premiers temps, cultivant la mystification comme un art (et dont il conviendrait de requestionner l'ingénuité ou la naïveté).

Donc : a) une approche authentiquement documentaire ne nie pas le fait qu'un documentariste digne de ce nom, aussi honnête qu'il soit envers la réalité qu'il filme, restitue un "point de vue subjectif» " ${ }^{4}$, même dans le cas où il aurait la naïve prétention 
à l'objectivité. Subjectivité accentuée par le travail concomitant ou subséquent sur le matériau filmique, même si la caméra entre ses mains n'est pas forcément une boîte à malice; b) elle ne relève pas non plus d'un phénomène de "mystification malicieuse» évoquant les pseudo-documentaires des émissions télévisées racoleuses ou même les fausses "actualités" du cinéma des premiers temps; c) on n'est pas non plus en présence d'un travail de "reconstitution exhaustive» d'une "réalité» factice évoquant le cinéma de fiction historique, biographique, etc.; d) on n'est pas non plus en présence d'un travail de "reconstitution plus subtile» qui se disqualifie du seul fait qu'il date déjà à la seule étape de la prise de vues, qu'il est décalé par rapport à la "réalité" qu'il prétend restituer. Le documentaire véritable, ainsi défini par la négative, empreint d'une subjectivité consciente, ne tend pas tant à «faire vrai», récusant d'entrée cet aphorisme liminaire, qu’à proposer une rencontre avec le spectateur, qu'à risquer une expérience de "vérité» entre deux subjectivités. Ce détour était nécessaire avant d'aborder ce qui suit.

Les mots (maux) de la tribu (l'attribut)

Ainsi, pour de mauvaises raisons, dont la mauvaise conscience de certains analystes qui, à la manière des jansénistes, pouvaient difficilement s'accomoder du fait qu'il y a forcément un "travail» (et du plaisir) derrière les images animées, on en est arrivé en à dire "tout est fiction", donc que tout s'équivaut, que tout s'annule, documentaire et fiction ou inversement. Comme l'aphorisme disant que le cinéma sorte toujours perdant à vouloir faire vrai, cette simplification correspond à dire tout et rien du tout à la fois, puisque cela parle à la fois de tout et de rien. La confusion des "genres" qui en découle, due à un simple dérapage d'un niveau de langage à un autre, serait sans conséquence si elle ne trouvait écho dans un fait de société plus vaste, idéologique, dont les répercussions se font sentir sur la production documentaire même.

Lapproche craintive des organismes subventionneurs jointe aux phénomènes de censure des institutions et d'autocensure de la part des cinéastes ont fait le reste, avec le résultat que les documentaristes ont vu leur champ d'intervention rétrécir comme peau de chagrin. Pour survivre, il se sont crus pour la plupart 
dans l'obligation de faire des concessions à la fiction (au sens où ce mot est entendu par le commun des mortels - hors les cercles d'inités de la tribu), au point où les institutions et les organismes subventionneurs, en vertu d'un contrat contre-nature, exigent maintenant la scénarisation des projets de films documentaires, selon le modèle du cinéma de fiction le plus répandu.

Dans la foulée de ce dérapage, au nom d'une commodité langagière, toutes les formes de compromis impliquant simultanément une volonté documentaire et un désir de fiction se voient ramenées sous le vocable réducteur de la "docu-fiction". Ce phénomène de "pollinisation" ${ }^{5}$ revêt pourtant des modalités diverses, composant une mosaïque narrative et stylistique d'une grande richesse qui retient périodiquement l'attention, et il constitue l'un des axes qui nous permettent le mieux de cerner la mouvance du cinéma, par-delà le fait que les images animées quelles qu'elles soient, tout comme les mots, «fictionnalisent" (ou "fictionnent") en quelque sorte la "réalité».

Ces tentatives de dépassement des clivages traditionnels entre le film documentaire et le film de fiction recouvrent donc un ensemble de pratiques significatives qui sont peut-être ellesmêmes révélatrices des maux de "l'attribut" (la fiction/le documentaire?), lorsqu'elles ne constituent pas une tendance prometteuse pour le futur, en suscitant possiblement l'émergence de nouvelles façons de cerner l'insaisissable. Après un premier mouvement durant les années 60 au cours duquel des fictions furent "visitées" d'une façon fertile par le cinéma direct, dans une sorte de respect de la tradition documentaire et de désir de concevoir un nouveau type de fiction qui ne serait pas bêtement calqué sur le modèle américain, finalement imposé par les institutions au cours de la décennie suivante, documentaire et fiction se sont à nouveau mutuellementent courtisés au tournant des années 80 , donnant lieu à une "pollinisation croisée», et le phénomène a touché particulièrement le cinéma québécois. Amorcé par le magnifique coup d'envoi de Jacques et Novembre (1984), côté fiction, et d'autres films comme La Turlute des années dures (1983), côté documentaire, cette nouvelle vague a donné lieu à un ensemble d'expérimentations, allant du meilleur au pire. Avec le recul, on s'aperçoit que, si elle est justifiée d'un point vue 
théorique et éthique, la pratique de la simple «juxtaposition" fait souvent problème (dramatiquement, les personnages et les situations fictives n'ont pas le temps ni l'espace pour s'imposer, et, loin de la soutenir, cette fiction distrait de la visée documentaire), alors que celle qui tend à réaliser une "fusion", une osmose des procédés et des genres, éminemment discutable dans l'optique documentariste (posant essentiellement un problème éthique de crédibilité), semble la plus riche de promesses pour le renouvellement des structures du récit, du moins pour le cinéma de fiction ${ }^{6}$ et pour la démarche apparentée à "l'essai» (voir plus loin) qui suppose d'emblée une très forte implication personnelle de l'auteur, une subjectivité assumée nettement plus prégnante que dans ce qu'il est convenu d'appeler le documentaire. À l'heure où le cinéma québécois est devenu un cinéma de producteurs, ce butinage d'une genre à l'autre a ralenti au tournant des années 90, au profit d'une autre type d'échanges entre le cinéma et la vidéo, qui dépasse de loin la simple question des supports (pellicule/ bande magnétique), comme l'annonçait déjà aussi Jacques et Novembre, pour travailler davantage à l'intérieur d'un même "genre», d'une même famille d'images, et pour céder la place incidemment à de plus en plus de "vraies" fictions, et à de moins en moins de "faux" documentaires.

Un jeu de mots aussi facile qu'il semble incontournable, qui nous ramène à la case départ et qui nous fournit l'occasion de revenir sur la terminologie employée pour tenter de cerner les modalités du phénomène, dont les expressions génériques docudrama et docu-fiction employées respectivement dans les milieux anglophone et francophone, pour en arriver à une classification plus fine, en gardant à l'esprit qu'il s'agit d'une avancée sur un terrain miné et qu'un film correspond rarement de part en part à une seule de ces expressions, à une seule des modalités qu'elles désignent. Aussi, les exemples avancés ici, puisés dans le cinéma québécois, sont choisis en fonction de la modalité dominante qui les caractérisent :

1. DoCu-Drame : cette expression dérivée de l'anglais "docu-drama" devrait être réservée à désigner un film qui propose une "reconstitution fictive» de faits divers réels, comme s'ils étaient captés et restitués "au 1 cr degré», voire "objectivement», et 
qui se donne à lire comme tel. Il vise à illustrer une situation donnée, un fait vécu antérieurement, ses sources de documentation peuvent être diverses. Il peut s'apparenter aussi bien au documentaire qu'au cinéma de fiction.

Exemples: Une guerre dans mon jardin (1985) de Diane Létourneau. Mais aussi des films fort différents comme L'Affaire Coffin (1979) de Jean-Claude Labrecque s'en rapprochent singulièrement.

2. DOCU-FICTION : cette expression devrait être réservée à désigner un film qui présente une "juxtaposition" (plutôt qu'une fusion), un assemblage grossier des "genres", séparés et aisément repérables. Si parfois il fonctionne mal au plan structurel, en

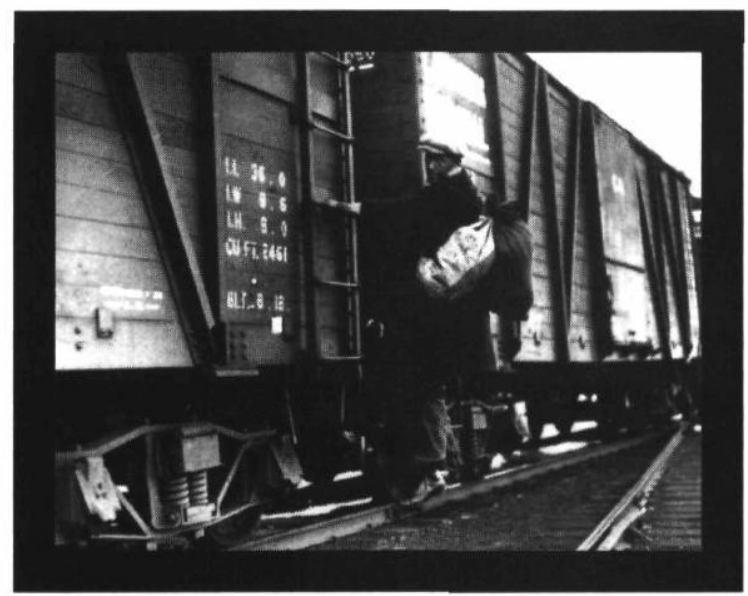

LA TURLUTE DES ANNÉS DURES

de Richard Boutet (1983)

Coll. Cinémathèque québécoise

évoquant le "raboutage», cet assemblage offre l'avantage de ne pas duper le spectateur quant à l'utilisation de plages documentaires et de passages qui relèvent ouvertement de la fiction. (L'article "le" conviendrait pour les cas où la part documentaire l'emporte sur la fiction; inversement, l'article «la» serait de mise dans les cas où la fiction est prééminente.)

Exemples : Caffé Italia, Montréal (1985) de Paul Tana, qui offre 
la particularité d'une forme trop éclatée, qui ne répond pas vraiment à une nécessité; Pourquoi l'étrange Monsieur Zolock sintéressait-il tant à la bande dessinée? (1983) d'Yves Simoneau, ou Le Million tout-puissant (1985) de Michel Moreau, qui fonctionnent tous deux sur le principe de l'enquête fictive alors que le contenu se veut documentaire, le film de Moreau étant plus symptomatique d'un malaise que novateur, où la fiction en arrive à désamorcer, voire à étouffer la visée documentaire; Le Dernier Glacier (1985) de Jacques Leduc qui, à travers une fiction assez lourde, développe son propos sur le mode métaphorique (éclatement du couple/mort de la ville), et qui relève aussi de la fiction- «vérité»; Mémoire battante (1983) d'Arthur Lamothe qui se présente comme un documentaire mâtiné de séquences dramatiques avec des comédiens professionnels, avec en plus une intervention personnelle du réalisateur (qui le fait participer aussi de l'essai); La Turlute des années dures et La Guerre oubliée (1987) de Richard Boutet se rattachent aussi à cette catégorie, tout en offrant la particularité, dans le premier cas, d'une mise en situation des personnages qui s'apparente par moments à la mise en scène, et dans le second cas, d'un dispositif scénique ingénieux d'économie, visant non pas tant à une «reconstitution" qu'à une suggestion d'ordre dramatique de certains faits historiques.

3. DOCU-FICTION AUTOBIOGRAPHIQUe : viserait à définir une "démarche mi-fictive, mi-documentaire concernant l'auteur même du film", démarche qui fait appel à la juxtaposition de divers procédés techniques ou narratifs, voire à leur fusion.

Exemples : tout en s'en distinguant chacun à sa façon, des films comme Journal inachevé (1982) de Marilù Mallet ou Celui qui voit les heures (1985) de Pierre Goupil se rattachent à la fiction-«vérité», dans la mesure où les "acteurs" sont «à la fois» à l'écran eux-mêmes et des personnages, et non de simples personnages. Par ailleurs, ils relèvent de cette appellation imparfaite qu'est la docu-fiction autobiographique, dans la mesure où il $\mathrm{y} a$ tantôt fusion des rôles et des genres, mais sans qu'ils soient clairement révélés au spectateur, tantôt simple juxtaposition de divers éléments. Cette approche évoque tantôt la "technique mixte» des arts plastiques, tantôt le collage. 
4. Fiction DOCUMENTÉE : expression déjà consacrée ${ }^{7}$ désignant une fiction inspirée étroitement de faits réels et filmée d'une façon quasi documentaire (techniques, méthodes de tournage, etc.), mais sans prétendre à une reconstitution "objective». "Fusion" des genres réalisée, mais "dévoilée au spectateur" et clairement perceptible au niveau de l'énonciation, sans exclure pour autant l'intensité dramatique. De plus, il peut proposer un questionnement sur sa propre pratique, sur sa démarche, en misant sur certains effets de distanciation (le glissement d'un même personnage d'un niveau à un autre, la rétroaction, etc.), afin de susciter la réflexion et l'analyse chez le spectateur.

Exemples : Les Ordres (1974) de Michel Brault, qui s'est imposé comme le modèle ${ }^{8}$ du genre; et partiellement, Mourir à tue-tête (1979) d'Anne-Claire Poirier, qui joue sur plusieurs registres.

5. "DOCUMENTAIRE" FICTIF (les guillemets sont importants, s'il est fictif au sens courant du terme, il n'a pas d'existence en tant que documentaire) : cette expression désignerait une fiction qui a l'apparence confondante du documentaire, qui «induit ou qui tente sciemment d'induire le spectateur en erreur", soit pour le faire réfléchir en bout de ligne, soit pour simplement lui faire prendre la proie pour l'ombre.

Exemple : L'Homme renversé (1986) d'Yves Dion, avec la précision que la démarche trompeuse ici, qui d'ailleurs joue sur plusieurs tableaux à la fois, fait ultimement réfléchir le spectateur sur les moyens dont dispose le cinéma. (Néanmoins, il est ahurissant de constater à quel point certains spectateurs supposément articulés n'arrivent pas à distinguer clairement ses divers niveaux de fabrication, ni l'intentionnalité qui les sous-tend.)

6. FICTION DOCUMENTARISÉE : viserait à désigner une démarche dont la principale caractéristique, par rapport à l'optique qui est la nôtre ici, est l'utilisation de lieux réels simplement comme "décors" (qu'ils aient une fonction signifiante, symbolique, ou autre), comme faire-valoir en quelque sorte à un personnage purement fictif. En d'autres termes, elle viserait à désigner le recours aux éléments documentaires du "second plan" (voir plus loin) tout en la distinguant de la démarche de "fiction-vérité". 
Exemple : On retrouve dans cette catégorie d'innombrables films de fiction, comme Les Matins infidèles (1989) de Jean Beaudry et François Bouvier, où les personnages sont brièvement intégrés à la réalité du marathon de Montréal, etc. Certains films identifiés à d'autres catégories illustrent aussi partiellement ce procédé, comme Le Dernier Glacier de Jacques Leduc.

7. "FICTION-VÉRITÉ» (les guillemets sont importants, vu l'opposition de nature contenue dans l'expression) : par allusion au bref courant du "cinéma-vérité" du début des années 60 dans le secteur documentaire, cette expression viserait à désigner une "démarche de fiction» qui a recours à certains éléments de la

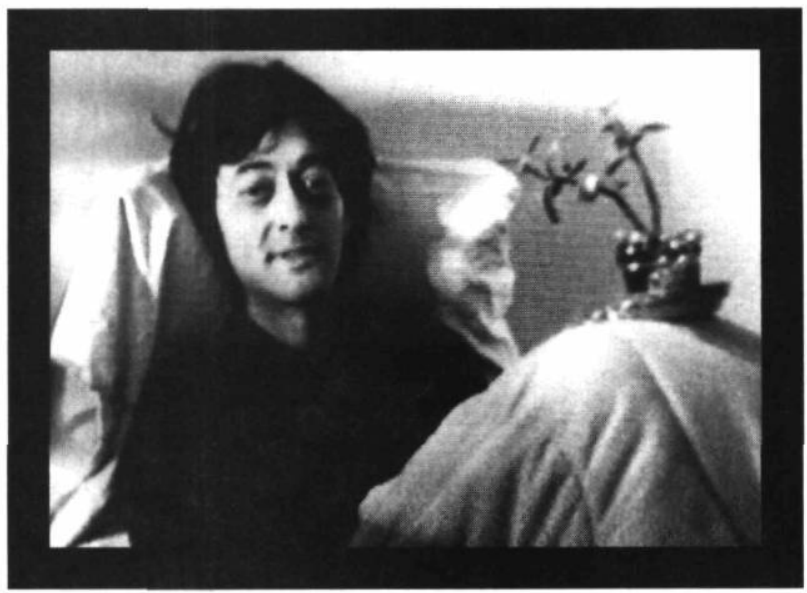

JACQUES ET NOVEMBRE

de Jean Beaudry et François Bouvier (1984)

Coll. Cinémathèque québécoise

démarche documentaire pour renforcer son "climat d'authenticité», notamment en misant sur la concordance entre le personnage à l'écran et la personne dans la vie chargée de l'incarner, le plus souvent un non-acteur ou un acteur non professionnel, et les faisant évoluer dans des situations et des lieux réels, etc. Trait dominant de plusieurs films de fiction importants des années 60 , représentatifs de cette fusion possible entre les moyens du 
documentaire (direct) et ceux de la fiction.

Exemples : Le Chat dans le sac (1963) de Gilles Groulx, Entre la mer et l'eau douce (1967) de Michel Brault, ou À tout prendre (1963) de Claude Jutra et Prologue (1969) de Robin Spry (partiel).

Cette catégorie, particulièrement riche de virtualités, appelle de nombreuses nuances. Par exemple, un film comme Jacques et Novembre de Jean Beaudry et François Bouvier, composé en grande partie d'un journal intime enregistré sur vidéo, ce qui "génère un effet de réel fascinant, que vient accentuer le jeu sans fard des comédiens" ${ }^{10}$, relève à la fois de cette catégorie et partiellement de celle du "documentaire" fictif défini plus loin. Alors qu'un film comme Journal inachevé de Marilù Mallet procède à la fois de cette catégorie et de la docu-fiction autobiographique.

Cette catégorie se distingue de la fiction documentarisée, caractérisée par la seule utilisation de lieux réels comme "décors». Par exemple, un film comme L'Hiver bleu (1979) d'André Blanchard procéderait à la fois, semble-t-il, de la "fiction-vérité" et de la fiction documentarisée.

8. DOCUMENTAIRE FICTIONNALISÉ : cette expression viserait à définir un film qui affiche une volonté résolument documentaire, mais qui «intègre à son propos des séquences de fiction", voire qui mêle à la fois des témoignages authentiques, live, à des "témoignages" fabriqués de toutes pièces pour les besoins de la cause. Il y a fusion des divers éléments ou à tout le moins une juxtaposition telle des divers éléments et procédés qu'elle tend à gommer, à occulter leurs marques de différenciation. Porte ouverte à tous les abus possibles, cette modalité, qui peut donner lieu parfois à des résultats étonnants, repose en définitive sur l'honnêteté du cinéaste.

Exemple : La Peau et les os (1989) de Johanne Prégent où les témoignages "fictifs" sont en quelque sorte authentifiés par des extraits documentaires saisissants, et qui lorgne aussi du côté de la docu-fiction par des séquences théâtrales.

9. ESSAI : désignerait une démarche, voire une façon de mener une "enquête", de type documentaire, sur un ton éminemment personnel, au point où cette subjectivité exacerbée (différente de la subjectivité sous-jacente à tout image) conduit à

Les Mots de la tribu 
un résultat inclassable, entre le cinéma documentaire et le cinéma de fiction ${ }^{11}$.

Exemple : Voyage en Amérique avec un cheval emprunté (1987) de Jean Chabot, Alias Will James (1988) de Jacques Godbout.

\section{TABleaU RECAPitulatif DES MOdalités \\ DE LA «POLLINISATION"}

1. DOCU-DRAME (narration au premier degré)

2. Docu-FICTION (juxtaposition évidente)

3. Docu-fiction autobiographique (projection de la vie de l'auteur)

4. Fiction DOCUMENTÉE (fusion, mais dévoilée au spectateur)

5. DOCUMENTAIRE FICTIF (faux documentaire)

6. Fiction documentaRisÉE (lieux réels comme «décors» : le rôle du second plan)

7. «FICTION-VÉRITÉ»(projection personne/personnage, approche de type documentaire)

8. Documentaire fictionnalisé (fusion ou juxtaposition qui tend à occulter les marques de différenciation entre les passages documentaires et les séquences de fiction fabriquées expressément)

9. EsSAI (démarche de type documentaire sur un ton éminemment personnel)

Bien sûr, en plus d'être fragiles, ces catégories ne sont d'aucun intérêt pour elles-mêmes, les distinctions qu'elles suggèrent, perceptibles par tout spectateur articulé qui fréquente les "films", visent avant tout, en focalisant sur ce phénomène de pollinisation des pratiques, à permettre de mieux évaluer les modalités de la "fictionnalisation" du cinéma. 
Au contraire du cinéma de fiction, il n'est pas sûr que le cinéma documentaire trouve toujours son compte dans ces effets de transgression par le simple amalgame (pourtant justifié du seul point de vue théorique), par la fusion ou le détournement (qui peuvent atteindre à une efficacité redoutable) de méthodes et de techniques identifiées, surtout quand la fiction vient se fondre littéralement à la démarche documentaire (comme si elle ne "fictionnalisait" pas déjà suffisamment dans l'absolu), dans une attitude de mimétisme, au point de lui faire perdre son intégrité et de poser un problème de perception au spectateur, en le plaçant dans une position vaseuse, alors qu'il lui arrive déjà de confondre fréquemment le réel et l'imaginaire, la vie et l'univers des signes. Car, en définitive, c'est lui qui est concerné, ce nouveau "primitif" à travers lequel se profile peut-être la tribu «zappeuse» du troisième type. Mais, si certaines productions hybrides ne sont pas sans évoquer la pratique du zapping télévisuel, l'exploration de nouvelles structures du récit, qui accompagne ces effets de transgression et qui s'effectue sur le mode d'une forte subjectivité assumée, par-delà les stratégies à court terme et les effets de mode, pourrait ouvrir de nouvelles perspectives, comme le laissent déjà percevoir certains films. Alors cette révision des enjeux esthétiques ne conduira pas à une simple dérive idéologique.

\section{Cinéma et Histoire (histoires)}

Ces fréquentations du documentaire et de la fiction sont symptomatiques d'une époque et elles ne sont pas sans faire référence à la représentation de l'Histoire actuelle ${ }^{12}$ ou passée, plus ou moins "documentarisée» ou "fictionnalisée», telle qu'elle se manifeste sur l'écran ou à la télévision, à la façon même dont nous sont montrés maintenant certains "hauts faits d'armes" (comme le spectacle fabriqué et aseptisé de la guerre du Golfe, sur l'ensemble des réseaux de la télévision mondiale ${ }^{13}$ ). Ce singulier travail sur la représentation qui correspond à rien de moins qu'à un détournement de l'Histoire en train de se faire et de s'écrire (par l'écrit et par l'image) survient à une époque où l'Histoire n'est à peu près plus enseignée dans les écoles, en Occident, sous le prétexte de l'objectivité, à une époque où, de plus, la liberté d'expression bat en brèche un peu partout, sous le prétexte d'éviter les sujets tabous. De fait, cette politique de 
l'autruche favorise l'établissement de chasses gardées du savoir qui en arrivent à constituer en mythes des faits historiques qui gagneraient à être librement discutés, et elle correspond à un refus de reconnaitre officiellement l'existence d'une vision inévitablement interprétative, donc subjective, de l'Histoire. Celle-ci étant forcément jalonnée de luttes, de conquêtes et de défaites, le point de vue du vainqueur s'opposant à celui du vaincu, quelle Histoire privilégier? L'Histoire du Québec ou du Canada? l'Histoire de l'Irlande ou de l'Angleterre? le point de vue arabe ou le point de vue sioniste? Consentir à un tel choix, proposer un point de vue ou une piste de lecture des événements revient sans doute, sous un angle théorique, à "fictionnaliser" l'Histoire. D'un côté comme de l'autre. Dès lors, au nom de quel principe en privilégier un seul?. Ce refus borné conduit au résultat que le jeune spectateur d'aujourd'hui, privé de repères, constitue ou croit constituer sa propre vision de l'Histoire, qui lui est de fait imposée à son insu à travers les «histoires» que lui racontent la télévision ou le cinéma dominant.

C'est dans ce contexte que la démarche d'un Marc Ferro ${ }^{14}$ apparaît intéressante, qui propose de multiples façons de «lire» les films afin d'en extirper des significations historiques. Illustrée à travers la série télévisée Histoire parallèle, comparant les actualités allemandes et alliées de la Seconde Guerre mondiale, son approche fondée sur l'intuition propose "la critique publique des sources et de leurs manipulations", ainsi que "des images parfaitement antithétiques représentatives des camps opposés». Partisan de la multiplication des "regards croisés»" ${ }^{15}$, il se trouve à favoriser une approche moins myope du "texte" (filmique), pour déborder sur d'autres aspects comme l'idéologie ou ultimement le mythe. Au lieu du seul point de vue officiel univoque, inévitablement mensonger de nature, il s'agit, d'une façon plus générale, de faire état des divers points de vue et traitements produits par diverses instances (individus, groupes, pays, etc.) à propos d'un même événement, et partant, de faire état "publiquement" des multiples lectures possibles qui en résultent. Mais, selon cette même stratégie, relativement sage ici dans son approche comparatiste, on pourrait aussi proposer des images de camps alliés ou opposés, à propos de situations et d'événements analogues même 
éloignés dans le temps et l'espace, et dynamiser ainsi ce travail sur la mémoire, rendant à l'Histoire sa fonction première qui est de favoriser une meilleure compréhension du présent.

Certes, nombreux sont les historiens à voir dans le cinéma une source d'informations douteuse et les théoriciens du cinéma à récuser toute approche intuitive. Néanmoins, par-delà ces barrages, l'idée est en train de faire son chemin que les films, documentaires ou de fiction, nous renseignent, comme ils peuvent aussi mentir ou contredire le consensus social, sur l'évolution des mentalités et des rapports sociaux, lorsqu'ils ne traduisent pas des attentes collectives latentes. Interprétés et mis en relation avec d'autres éléments (dont le contexte de production et de diffusion, au sens large), ils peuvent donc s'avérer être des révélateurs de la société passée ou actuelle, et plus particulièrement de son refoulé social.

Bien évidemment, cette confrontation de points de vue opposés ou complémentaires n'a que l'apparence de l'objectivité, et elle a justement le mérite de faire voler en éclats ce mythe, cette tarte à la crème servie régulièrement par les groupes de pression et les instances au pouvoir pour imposer leur propre point de vue à travers des «images sous surveillance». Et il peut même en résulter des rapprochements d'images "scandaleux».

En partant du principe que rien n'est tabou dans l'Histoire, qu'aucune censure, audiovisuelle ou autre, ne peut se justifier, qu'aucun groupe ne peut constituer de chasses gardées du savoir, il n'y aurait de fait rien de scandaleux dans des rapprochements de ce type, si ce n'est la pénible réalité historique à laquelle ils font référence. Et, à 40 ou 50 ans de distance, l'image d'un Palestinien estropié à vie ou d'un Noir brûlé vif ne deviendrait pas plus "fictionnelle" ni moins "historique» que certaines images du passé. Mais, sans vouloir établir des équivalences simplistes, comme celle visant à justifier la guerre du Golfe (Hussein=Hitler), sous leur apparente objectivité, ce rapprochement et ce transfert de situations semblables, par le travail d'actualisation qui les sous-tend, seraient forcément porteurs d'un point de vue.

Plus qu'un simple procédé de montage, ce travail sur la mémoire, dynamisé par les moyens du cinéma et de l'audiovisuel, 
qui exigerait bien évidemment d'être contextualisé puisqu'il concerne des époques et des lieux différents, permettrait à l'Histoire de refaire surface pour une meilleure compréhension du présent qui ne corresponde pas à une perception univoque ou à courte vue...

Ce détour (provocateur? mais l'est-il plus que la "fiction" de la guerre du Golfe?) sur le pouvoir des images, leur manipulation, leur utilisation et leur questionnement nous ramène à notre point de départ. Dans la mesure où elles n'auront pas simplement cédé à la fonction distrayante du spectacle facile et vite fait de la télévision, ni cédé à son vertige du vide comblé par le babillage des "têtes parlantes", ces expérimentations hybrides en matière de cinéma entre le documentaire et la fiction auront certes contribué à agrandir le champ du "dicible filmique" en favorisant "l'amplification des signes» évoquée par le cinéaste Michel Moreau, notamment en légitimant le travail sur le montage. Mais, en même temps, elles auront favorisé la déroute du spectateur, ce nouveau "primitif" devenu le siège et l'enjeu d'un rituel au cours duquel des concepts théoriques et des propositions audiovisuelles concrètes lui sont décochés comme autant de flèches empoisonnées (même pour décrire le fonctionnement relativement simple du cinéma), au point où l'attribut de substitution en arrive à jouer le rôle de l'arbre qui lui masque la forêt, une forêt de signes contradictoires qui lui sont devenus incompréhensibles. Tant et si bien que ce spectateur, ce nouveau "primitif" se voit aujourd'hui manipulé au point de confondre souvent le réel et l'imaginaire, la vie et l'univers des signes, peu importe d'ailleurs le canal qu'ils empruntent maintenant, cinéma ou télévision.

En effet, pour lui, le glissement sémantique des mots documentaire et fiction s'accompagne d'un semblable mouvement des réalités qu'elles désignent puisque, comme l'ont démontré la représentation de la guerre du Golfe, procédant d'une mise en scène époustouflante, et le débarquement d'opérette en Somalie, "Il est clair que rien n'empêche plus désormais de mettre en scène la réalité pour en projeter les images» ${ }^{16}$. Le mythe est souverain. 


\section{NOTES}

1 Stéphane Mallarmé, "Le tombeau d'Edgar Poe", in Poésies. Hommages et tombeaux (Paris : NRF, 1920).

2 Cette affirmation n'est pas aussi farfelue qu'il semble. Le pionnier québécois L.E. Ouimet avait en son temps trouvé le moyen de contourner les foudres de l'Église catholique et ses menaces d'excommunication en vendant des bonbons à la porte de son établissement, achat qui permettait au client-spectateur d'assister "gratuitement" à la représentation de vues animées qui suivaient, autorisant du même coup L.E. Ouimet à exploiter son "théâtre" (son commerce) le dimanche, jour alors officiellement sacré au Canada. Sans ce subterfuge passager, la mine d'or de ce pionnier se serait rapidement épuisée, au profit de ses concurrents anglophones non catholiques. Aujourd'hui, empêtrés dans leurs contradictions (exacerbées par l'explosion des clubs vidéos), certains réseaux d'exploitation cinématographique, et non des moindres, ne subsistent que grâce à la vente du "pop corn" et autres cochonneries dans leurs salles.

3 Tout en reconnaissant, pour paraphraser Edgar Morin, que le cinéma documentaire camoufle son imaginaire, et partant sa fiction, alors que le cinéma de fiction porte sa vérité dans son imaginaire même, les expressions cinéma documentaire et cinéma de fiction sont employées ici dans leur sens courant pour définir des pratiques universellement reconnues.

4 Voir notre texte, "Pour en finir avec le mythe de l'objectivité, ou le droit à la subjectivité assumée", 24 Images no 46 (1989).

5 Terme avancé pour la première fois dans notre ouvrage L'Aventure du cinéma direct (Paris : Seghers, 1974). On emploie aussi indifféremment les termes hybridation et métissage. Pourtant, l'anthropomorphisme du mot métissage pourrait faire problème : il désigne un croisement, un mélange de races. Dès lors, par-delà l'implication de l'auteur et du spectateur, comment oser défendre la "pureté" d'une démarche documentaire ou de fiction, sans être soupçonné de racisme, ou au contraire affirmer sa sympathie pour le métissage, sans s'attirer la colère des tenants de la "politically correctness" qui semblent s'y opposer? Les termes hybridation et pollinisation échappent à cette connotation, mais le premier terme évoque un croisement "fécond" entre espèces (ce qui n'est pas toujours le cas entre fiction et documentaire), alors que le second terme offre l'avantage d'évoquer en outre la possibilité d'un mouvement "croisé" (rejoignant les "regards croisés» de Marc Ferro)!

6 Comme on pouvait déjà le percevoir au début des années 70. Voir "Quelques perspectives du cinéma direct", in L'Aventure du cinéma direct, ibid., pp. 311 et suivantes.

7 Voir "Cinéma direct", in Michel Coulombe et Marcel Jean (direction), Le Dictionnaire du cinéma québécois (Montréal : Boréal, 1991).

8 Voir notre ouvrage Les Ordres - Un film de Michel Brault (Montréal : L'Aurore, 1975).

9 Tel qu'il est abordé par Marie-Claude Bénard dans «Le Décalogue : le rôle du second plan", CinémAction no 65 (1992). Elle reprend l'idée que la pratique du tournage en extérieurs réels, hérité de la pratique documentaire du cinéma direct, a contribué à modifier la façon de faire des films de fiction, leur écriture et leur contenu, et elle avance que cette pratique va jusqu'à faire de certains films de fiction des documents sur le "décor" (terme ambigu désignant le "lieu de tournage», sur le terrain) qu'elle identifie au "second plan" : "celui de l'espace qui accompagne l'action". Elle précise : "Hors studios, le second plan présente un état des lieux, il est porteur d'usages, et révélateur de mentalités socialement et historiquement datées." Certes, "l'authenticité" de ce fond visuel est à questionner constamment, puisqu'il peut être l'objet de diverses 
manipulations (renvoyant à des "représentations subjectives de l'espace habité»), ne serait-ce qu'au seul niveau du montage qui peut en restituer une géographie autre. Mais ces manipulations spatiales peuvent aussi «être volontairement limitées (par le cinéaste) dans le respect de la convention réalisten.

10 Marcel Jean, Le Cinéma québécois (Montréal : Boréal, 1991).

11 Terme avancé par Michel Euvrard dans une optique plus large visant l'ensemble des phénomènes du métissage, in Copie Zéro no 30 (1986).

12 Même si pour certains historiens, l'Histoire récente n'est pas à proprement parler de l'Histoire.

13 Lire à ce sujet : "Médias, mensonges et démocratie", Le Monde diplomatique no 14 (février 1992). À l'occasion de la couverture médiatique de la guerre du Golfe, on y avance l'idée que "la mise en spectacle des informations, telle que l'a réussie la chaîne $\mathrm{CNN}$, n'est qu'une étape d'une longue dérive qui tend à effacer la frontière entre la réalité et la fiction".

14 En rendant hommage à Marc Ferro, la revue CinémAction a consacré un numéro à ce phénomène de la rencontre entre le cinéma et l'Histoire, nous inspirant ces lignes.

15 Selon l'expression de François Garçon, in CinémAction. Voir à ce sujet Pierre Sorlin et François Garçon, "De Braudel à "Histoire parallèle", avec Marc Ferro", CinémAction n' 65 (1992).

16 Voir l'article de Paul Warren, "Images américaines", Le Devoir (16 janvier 1993), qui se penche sur ces rapports de la fiction et du documentaire dans le cinéma américain. 